\title{
Le temps signifiant et le Moyen Âge français
}

\section{Weather-meaning in the French Middle Ages}

\author{
Andrea Tarnowski \\ Dartmouth College \\ andrea.tarnowski@dartmouth.edu
}

\begin{abstract}
An analysis of "weather events" and their meaning in works of French medieval literature - La Chanson de Roland, Le Chevalier au lion, Le Roman de la rose, Le Livre du Cuer d'amours espris and Le Debat d'entre le gris et le noir - finds different forms of interaction between the outside world and human beings. Whether a connection between man and nature is mediated by God, set by the human arrangement of or incursion into a natural setting, or left so loose as to suggest nature's indifference to human witness, weather contributes to the picture.
\end{abstract}

Keywords: weather, environment, Le Debat d'entre le gris et le noir, La Chanson de Roland, Chrétien de Troyes, René d'Anjou

On débute par la fin et dans la discrétion, faisant appel à un texte peu connu de la fin du Moyen Âge qui ne subsiste qu'en deux manuscrits. Il s'agit du Debat d'entre le gris et le noir d'Aymon de Montfaucon, où deux jeunes chevaliers discutent de l'attitude à adopter face aux dames qu'ils aiment ; aucun événement météorologique n'intervient au cours de ces échanges. Le chevalier Gris espère toujours entrer dans les bonnes grâces d'une dame qui le traite avec rigueur. Le chevalier Noir n'espère plus - sa dame est morte - mais cela ne l'empêche pas de lui vouer un culte absolu. Les deux amis s'opposent en ce que l'un désire l'avenir et croit encore à la conclusion favorable de ses efforts ; l'autre, figé dans un deuil que son honneur veut perpétuel, ne peut qu'invoquer la mort. Le débat sur l'amour se termine sans se résoudre, et le Gris consterné par la rigidité du discours de son compagnon l'écoute se lamenter et l'observe s'endormir. 
La discussion conclue, l'insatisfaction infuse, il faut trouver moyen de clore le récit. Le jour point à l'horizon, le chevalier Noir dort toujours, et le chevalier Gris, peiné par la tristesse de son ami, éprouve en même temps l'envie de reprendre seul son chemin. Mais pour éviter que le départ du Gris ne paraisse déloyal, il convient qu'une force extérieure s'impose. Voilà que la météorologie vient en aide au Gris sous forme d'orage ; il est obligé de quitter le refuge buissonnier d'où il a veillé sur le sommeil du Noir :

Ung peu devant l'aube du jour,

De mon vert giste me party,

Car plus n'y peu faire sejour,

Pluye et gresle m'en departy,

Qui survindrent par tel party

Que je fuz contrainct de partir.

(vv. 1731-1736)

La pluie et la grêle fournissent une raison de se séparer ; le Gris n'est pas lui-même responsable de l'acte de partir. L'auteur crée un mécanisme narratif pour orienter le poème vers sa fin ; l'orage est un outil pour avancer l'action sans pour autant impliquer les désirs ou les intentions du chevalier partant. Ces quelques vers d'un texte relativement obscur du $\mathrm{XV}^{\mathrm{e}}$ siècle ${ }^{2}$ suggèrent que le temps y est un outil littéraire qui sert une structure, plutôt qu'un générateur de signification en soi. La pluie n'attriste pas, la grêle ne provoque pas la peur, ni à l'intérieur du récit, ni chez le lecteur. L'événement climatique affecte les mouvements du protagoniste, mais ne reflète ni ses idées ni sa qualité morale. La nature est un décor et non un signe.

Il n'en va pas ainsi dans des textes antérieurs, qui posent un lien de sens entre le monde physique, surtout quand il est modifié par le temps, et les hommes qui l'habitent. Le témoignage de La Chanson de Roland est précieux à cet égard. Un orage se déclenche lorsque les troupes de l'empereur Charlemagne se battent contre leurs ennemis sarrasins ; il est autrement plus puissant que la tombée de pluie qui sépare les deux chevaliers débatteurs quatre siècles plus tard. L'action de la bataille se passe en Espagne, dans un paysage immuable et peu hospitalier : roches, cols sinueux et aspect désertique. Les seules couleurs au tableau sont le vert de l'herbe et le rouge du sang qui coule dessus ; la clarté du jour et l'obscurité de la nuit se succèdent dans un cycle invariable et non détaillé. L'environnement ne " parle » donc pas, sinon pour signaler dureté et danger; il met en avant la charge des chevaux et les gestes violents des guerriers. Mais au milieu de la mêlée, alors que des deux côtés des hommes nombreux tombent et meurent, survient l'orage... en France. La météorologie se déplace donc par-delà les Pyrénées pour apporter un message aux Francs. Les hommes de

${ }^{1}$ Une édition du Débat entre le gris et le noir faite par Emma Cayley et Olivier Delsaux est en voie de préparation. Je remercie ces deux collègues généreux de m'avoir communiqué leur travail.

${ }^{2}$ Cayley et Delsaux fixent 1473 comme le terminus ad quem de ce texte. 
Charlemagne poursuivent leur combat, tandis que le temps communique à leurs compatriotes restés sur des terres d'empire :

En France éclate une prodigieuse tourmente :

tempêtes de vent et de tonnerre,

pluie et grêle exceptionnelles ;

la foudre tombe coup sur coup, maintes et maintes fois,

c'est, à vrai dire, un tremblement de terre :

de Saint-Michel-du-Péril jusqu'à Xanten,

de Besançon jusqu'au port de Wissant,

aucune maison dont une partie des murs ne s'affaisse.

Et, dès midi, le jour s'obscurcit :

aucune lumière sinon quand le ciel se fend.

Nul ne le voit qui ne s'en épouvante,

et plusieurs disent : "C'est la fin du monde,

et nous voici à la consommation des temps ».

(La Chanson de Roland, 1990, p. 121, laisse 110, vv. 1423-1435)

Tout le territoire franc vit l'intensité de la bataille, son horreur et son bouleversement, traduit en un phénomène de la nature et à l'échelle de chaque maison, chaque vie. L'orage se saisit des êtres humains, mais non ceux qui guerroient en Espagne ; il n'y a pas ici symbiose entre les acteurs et leur cadre. La seule raison d'être de la tourmente est de signifier, de faire en sorte que les hommes interprètent et comprennent un événement auquel ils n'ont pas un accès direct.

Comme tout message, l'orage est sujet à une lecture erronée. C'est le cas ici, lorsque les gens voient dans les foudres l'arrivée de l'Apocalypse. La catastrophe est totale, en effet. Mais le poète de La Chanson de Roland, ayant associé ces affres à la fin des temps pour en souligner la gravité, corrige l'interprétation :

\footnotetext{
${ }^{3}$ Le texte en ancien français, d'après l'édition d'Ian Short :

En France en ad mult merveillus turment :

Orez i ad de tuneire e de vent,

Pluie e gresilz desmesurëement

Chiedent i fuildres e menut e suvent,

Et terremoete ço i ad veirement :

De Seint Michel del Peril josqu'as Seinz,

Dés Besençun tresqu'al port de Guitsand,

Nen ad recét dunt del mur ne cravent.

Cuntre midi tenebres i ad granz:

N'i ad clartét se li ciels nen i fent.

Hume ne l'veit ki mult ne s'espoënt ;

Dïent plusor : 'C'est li definement,

La fin del secle ki nus est en present'.

(La Chanson de Roland, 1990, p. 120, laisse 110, vv. 1423-1435)
} 
et plusieurs disent : « C'est la fin du monde, et nous voici à la consommation des temps" " Ils ne savent pas, ils ne disent pas la vérité : c'est là le deuil universel pour la mort de Roland.

(La Chanson de Roland, 1990, p. 121, laisse 110, vv. 1434-1437) ${ }^{4}$

Cette mort est encore à venir dans la chronologie du poème, et l'orage est donc prophétique en même temps qu'il exprime le déchirement. Il est créé, «monté » par le scribe pour offrir l'image de la nature entière réagissant à des faits humains, le tout reflétant un cas singulier. Le temps porte une plénitude de sens dont le poète guide l'expression.

Si Dieu n'est pas explicitement mentionné dans ce passage du Roland, sa présence anime le paysage aussi bien que les combattants. La nature n'agit pas, et surtout ne signifie pas, en tant que force indépendante. Le deuxième événement météorologique du texte - météorologique au sens large, puisqu'il s'agit d'une suspension du cours du soleil - confirme cette notion. Après le combat où tombe Roland, le jour décline. Charlemagne sait qu'il lui faut de la lumière pour livrer bataille aux Sarrasins et venger les morts franques ; il prie donc le Seigneur d'arrêter le soleil pour lui. Et Dieu l'écoute : «Pour Charlemagne, Dieu fit un grand miracle, / car le soleil s'est arrêté, immobile » (La Chanson de Roland, 1990, p. 185, laisse 180, vv. 2458-2459) ${ }^{5}$. Transposant les termes de la tempête qui s'était abattue sur la France, où à midi il faisait nuit noire, le poème présente un paysage où la clarté du crépuscule affirme la relation entre l'empereur et Dieu, et en manifeste le sens. Dieu donne raison à Charlemagne contre ses ennemis ; l'univers entier prend son parti.

Presque deux cents ans après La Chanson de Roland, un roman de Chrétien de Troyes fait jouer la météorologie en initiant l'action de son récit-cadre, puis en développant la narration principale. Le Chevalier au lion met d'abord en scène Calogrenant, chevalier à la cour du roi Arthur. Calogrenant raconte que six ans plus tôt, à la recherche d'aventures - suivant en cela le penchant de tout chevalier - il a rencontré un rustre. Celui-ci, tout en disant qu'il ne connaissait rien aux aventures, lui parle d'une piste intéressante. Il s'agit d'un endroit où l'on trouve un ensemble de merveilles : une fontaine extraordinaire qui bout d'eau froide, un arbre si sublime qu'il n'en a jamais poussé d'aussi beau, un bassin mystérieux en fer suspendu à l'arbre ${ }^{6}$, et un perron

${ }^{4}$ Dïent plusor : 'C'est li definement, La fin del secle ki nus est en present'.

Icil ne l' sevent, ne dient veir nïent:

C'est li granz doels por la mort de Rollant.

(La Chanson de Roland, 1990, p. 120, laisse 110, vv. 1434-1437)

${ }^{5}$ " Pur Karlemagne fist Deus vertuz mult granz, / Car li soleilz est remés en estant ». (La Chanson de Roland, 1990, p. 184, laisse 180, vv. 2458-2459)

${ }^{6}$ Nous apprendrons que le matériau dont est fait le bassin est en fait l'or fin (Chrétien de Troyes, 1994, p. 74-75, vv. 417-418) ; le rustre est trop ignorant pour reconnaître des métaux précieux. 
dont le rustre dit qu'il est indescriptible - il s'avère plus tard que cette plate-forme est faite d'une seule émeraude posée sur quatre rubis. On note que la disposition des éléments de la scène a requis une intervention humaine, ou surhumaine. Le temps va intervenir lui aussi, mais de manière " planifié ». Le texte ne donne pas l'origine de ce tableau de clairière, mais il est évident qu'il est porteur de sens ; il a été arrangé pour signifier. La fontaine, le pin, le bassin et le perron semblent attendre. Le rustre explique à Calogrenant comment faire, lui prédisant que s'il s'empare du bassin, récupère de l'eau dans la fontaine et la déverse sur le perron, il sera témoin d'un orage d'une violence sans pareille, qui dévastera la forêt alentour (Chrétien de Troyes, 1994, p. 74-75, vv. 393-402). Toujours désireux de vivre une aventure, Calogrenant trouve la fontaine et s'exécute ; son action provoque l'orage :

$[\ldots]$ je vis le ciel si perturbé
que, de plus de quatorze points,
les éclairs me frappaient les yeux,
et les nuages jetaient, pêle-mêle,
de la neige, de la pluie, et de la grêle.
Il faisait un temps si mauvais et si violent
que je croyais bien que j'allais mourir
à cause de la foudre qui tombait autour de moi
et des arbres qui se brisaient.
(Chrétien de Troyes, 1994, p. 77, vv. $438-446)^{7}$

Une fois l'angoisse passée, il éprouve un certain plaisir d'avoir affronté cette épreuve ; mais Calogrenant n'a pas compris que la scène composée de la fontaine, et l'orage qu'il a causé, ne se résumaient pas à des spectacles vides de sens. Il s'en rend compte lorsqu'il voit un autre chevalier, seigneur du lieu, arriver soudain, furieux de ce que son bois ait été détruit. Le chevalier seigneur défie Calogrenant, et triomphe sur lui dans un combat singulier. Calogrenant s'en retourne penaud, déshonoré par sa défaite. Mais peut-être est-il également déshonoré d'avoir pensé tester sa vaillance dans l'absence d'un contexte probant. Il n'a aidé aucune demoiselle en détresse, ni ne s'est mesuré à aucun géant maléfique ; loin d'avoir mis sa hardiesse au profit d'une bonne cause, comme il se devait, il a commis une faute en empiétant sur le domaine d'un 
autre. Il a fait fi du code d'honneur qui est censé régler sa conduite, et il paie le prix d'avoir suscité l'orage sans justification.

Calogrenant raconte son histoire au début du Chevalier au lion, mais c'est Yvain le chevalier du titre - qui en est le héros. Lorsqu'il écoute son cousin relater son échec vis-à-vis du seigneur de la fontaine, Yvain déclare qu'il vengera sa honte (Chrétien de Troyes, 1994, p. 78-79, vv. 479-588). Il part seul pour racheter l'erreur de Calogrenant, projetant de suivre le même chemin et d'accomplir les mêmes gestes jusqu'à ce qu'il catalyse l'orage. Ce qu'il fait : l'expérience se reproduit à l'identique (Chrétien de Troyes, 1994, p. 98-99, vv. 798-804). Mais comme son action est fondée, puisqu'il défend la réputation d'un chevalier parent, il a raison du seigneur du domaine qui ressort dès que son bois est de nouveau détruit. Deux observations s'imposent : d'une part, le «message » de la fontaine et celui de la tourmente ne découlent pas de la nature, mais de l'art. Le groupement intentionnel autour de la fontaine, le bassin d'or et le perron d'émeraude à l'orée du bois, signalent le pouvoir de son possesseur. D'autre part, l'orage sur commande, systématiquement reproductible et qui survient à chaque fois que des actions précises humaines le provoquent, n'est pas un événement météorologique à proprement parler, encore moins le signe d'une relation particulière avec Dieu. C'est un temps signifiant, une lecture qui s'offre au déchiffrement.

Contrairement à La Chanson de Roland et au Chevalier au lion, le Roman de la rose de Guillaume de Lorris (vers 1240) propose un environnement physique fourmillant de détails concrets - même si l'expérience décrite a lieu en rêve. Le narrateur relate un songe dans lequel il se trouve dans la nature au mois de mai, puis élabore son image pour dire comment le temps de ce mois printanier se manifeste : les buissons et les haies revêtent de nouvelles feuilles; une profusion d'herbes et de fleurs blanches et bleues recouvrent la terre ; une grande assemblée d'oiseaux - le narrateur précise les noms des rossignols, des perroquets et des alouettes - se réunissent pour chanter leur joie et donc pour rehausser la beauté de la saison (Guillaume de Lorris, 1992, p. 44-45, vv. 50, 52, 63, 74, 77). Un autre type de précision ou de relief est apporté par le contraste tracé entre les bourgeonnements du mois de mai et la froide nudité de l'hiver :

Les bois, qui sont secs tant que dure l'hiver, retrouvent leur verdure. La terre $[\ldots]$ oublie l'indigence qu'elle a connue tout l'hiver. [...] Les oiseaux, qui se sont tus aussi longtemps qu'ils ont subi le froid et le mauvais temps d'hiver, sont en mai, avec le temps serein, si contents [...]. (Guillaume de Lorris, 1992, p. 45, vv. 53-55; 57-58; 67-71)

\footnotetext{
${ }^{8} \mathrm{Li}$ bois recuevrent lor verdure, Qui sont sec tant com yvers dure.

La terre $[\ldots]$

[...] oublie la povreté

Ou ele a tout l'iver esté.

[...]

Li oissel qui se sont teü
} 
La douceur de l'air et l'intensité des couleurs sont d'autant plus saisissables que leurs opposés sont aussi évoqués ; on comprend mieux les traits de la belle saison quand on y compare les mois hivernaux.

Suivant le cours d'une rivière qui constitue un élément clé de la nature bienveillante mise en scène (Guillaume de Lorris, 1992, p. 48-49, vv. 126-128), le narrateur accède à un endroit qui amplifie les plaisirs du printemps dans un contexte moins réel, ou plus clairement rêvé : il s'agit du jardin dans lequel se jouera l'action du roman, et où il rencontrera la rose dont il tombera amoureux. Si l'inventaire de l'environnement est déjà abondant lorsque le narrateur se promène, il foisonne lorsqu'il entre dans l'enceinte du jardin : les noms d'arbres, de fruits, d'oiseaux se succèdent et se multiplient, la fraîcheur et l'agrément du lieu sont soulignés. La règle des contrastes qui fait ressortir la signification de deux termes opposés s'applique ; le narrateur rencontre d'abord une enceinte peinte de figures qui représentent toutes sortes de vices, de bassesses, et de désagréments : haine, convoitise, vieillesse et pauvreté, entre autres (Guillaume de Lorris, 1992, p. 48-65, vv. 143-470). La présence de ces figures sur le mur extérieur prépare l'entrée du narrateur au jardin, dont tout ce qui pourrait ennuyer ou dépiter a bel et bien été exclu. Le jardin se présente donc comme une version encore plus belle et verdoyante du paysage où se trouvait le narrateur au début de son rêve. Et une fois cette perfection établie, elle restera intacte jusqu'à la fin du texte (inachevé, il est vrai). Même si la Nature est louée d'avoir créé ces lieux délectables (Guillaume de Lorris, 1992, p. 112-113, vv. 1429-1431), le changement inhérent à la nature ne semble pas les affecter : la beauté du paysage et du jardin du rêve est statique.

Quant à la présence de l'eau dans ce lieu idyllique, la rivière qui menait au jardin se métamorphose, à l'intérieur du mur de protection, en des ruisseaux éparpillés (Guillaume de Lorris, 1992, p. 108-111, vv. 1380-1390), puis en une fontaine. Cela ne fait qu'accentuer l'aspect immobile de l'éden ; à l'intérieur du jardin, l'eau surgit en permanence et ne mène nulle part. Il s'agit d'une fontaine abritée par un pin, comme chez Chrétien de Troyes. Cet ensemble pourrait paraître naturel : une source jaillissant d'une pierre, ombragée d'un arbre. Le mot « fontaine » en ancien français signalerait dans ce cas une eau vive sortant de terre. Mais « fontaine » peut également signifier une construction pour canaliser l'eau, ou un récipient pour la contenir, et cette acception suppose un travail humain ; quelqu'un doit œuvrer pour former l'objet. Les vers confirment que la fontaine dont il s'agit ici est une construction, une création; elle est assise sous l'arbre (Guillaume de Lorris, 1992, p. 112-113, v. 1431), ce qui suggère que l'on a fait apporter le marbre pour le poser à cet endroit. Mais surtout, la pierre de

Tant qu'il ont le froit eü

Et lou tens d'yver et frerin,

Sont en may pour le tens serin

Si lié $[\ldots]$.

(Guillaume de Lorris, 1992, p. 44, vv. 53-55 ; 57-58 ; 67-71) 
la fontaine est gravée, présentant une inscription (qui raconte l'histoire de Narcisse). La fontaine est donc une œuvre d'art, un objet façonné ; même si le texte affirme que c'est la nature qui « par grant mestrisse » l'a établie (Guillaume de Lorris, 1992, p. 112-113, v. 1430), des indices sont réunis pour démontrer le contraire. Le Roman de la rose offre au lecteur un monde du plus grand souci esthétique, hautement stylisé ; l'environnement du verger clos où évolue celui qui rêve est soigné, détaillé, poli. Le mystère du bassin suspendu au pin chez Chrétien de Troyes - objet qui attendait que chaque chevalier nouvellement arrivé accomplisse le geste qui déclencherait ses pouvoirs - est remplacé par « l'évidence » d'un monde axé sur le pouvoir du visuel. Le verger se reflète dans deux cristaux au fond de la fontaine. La nature, l'environnement, invitent à la contemplation dans ce verger-tableau. Chez Chrétien, l'homme affecte le paysage où il passe et cherche à le maîtriser. Chez Guillaume de Lorris, il le consomme simplement des yeux. La météorologie automatique de l'orage provoqué correspond, chez Chrétien, à la mission du chevalier en quête d'aventure. Le beau fixe du jardin de Guillaume de Lorris reflète lui aussi la préoccupation du jeune protagoniste, puisqu'il s'adonnera entièrement à l'amour. C'est l'idéal suprême et qui ne peut changer; cela ne veut pas dire que sa recherche est facile - loin de là - mais seulement que le but de l'amour est un constant, le seul digne de poursuite. Dans les deux cas, l'environnement fonde les expériences de ceux qui s'y meuvent, ou s'adapte à leurs objectifs. Le contexte physique du héros témoigne de ses désirs, et représente sa psychologie. Cela par opposition à La Chanson de Roland où le monde externe n'est pas une projection de l'esprit de l'homme, mais signe de la volonté divine.

Le Livre du cuer d'amours espris de René d'Anjou (1457) constitue à bien d'égards une réponse aux romans chevaleresques d'aventure et d'amour - une réponse dirions-nous ironique, car il rend hommage à ses prédécesseurs tout en démontant leurs mécanismes de fonction. S'agissant du temps qu'il peut faire, l'on ne peut manquer d'observer les modifications qu'apporte René à la scène de l'arbre, de la fontaine et du bassin développée dans le Chevalier au lion de Chrétien. Au lieu du pin robuste et dru, un tremble : « hault à merveilles » (René d'Anjou, 2003, p. 122), certes, mais aux feuilles délicates et frissonnantes. Au lieu d'un bassin en fin or, un récipient en laiton, suspendu à une chaîne de fer. Au lieu d'une fontaine faite d'une seule émeraude, un perron bien moins scintillant de marbre gris-brun. Le mystère et l'enchantement du roman de Chrétien se sont fanés. L'eau froide et pure de la fontaine du Chevalier au lion est devenue saumâtre chez René (René d'Anjou, 2003, p. 130) et qui plus est, le Cuer protagoniste l'imbibe sans s'apercevoir de sa qualité infecte ; il arrive à la fontaine lorsqu'il fait déjà nuit et doit tâtonner dans l'obscurité pour trouver à boire. La confusion et l'humiliation qui accompagnent le Cuer dans cette scène le démarquent du chevalier Yvain, grand, fort, actif et sûr de lui. Le paysage est là pour suggérer la distance entre les beaux idéaux chevaleresques d'aventure et d'amour et un vécu bien moins convaincant. A l'étape suivante de la scène, lorsque le Cuer déverse le bassin sur le perron après avoir bu et provoque un orage terrible accompagné 
de pluie et de grêle (René d'Anjou, 2003, p. 124), le souvenir du héros de Chrétien de Troyes colore encore la réaction du lecteur. Là où l'orage avait incité Yvain, lui-même indemne, à affronter un adversaire féroce, le Cuer réagit au temps violent en se retirant à l'abri sous le tremble. Et en attendant que l'orage se calme, il est trempé par la pluie, meurtri par la grêle, et s'endort tremblant, frissonnant du froid (René d'Anjou, 2003, p. 128). Ce triste spectacle augure mal de la suite, et en effet, après de longs périples et divers efforts, le Cuer devra renoncer à sa quête d'amour. L'épisode de Chrétien de Troyes semble avoir été vidé du suc de la croyance en un idéal, ou de témoigner plutôt d'un écart entre cette croyance et un résultat satisfaisant qui en découlerait. La minutie avec laquelle René reprend, puis remodèle Chrétien prouve que le temps et l'environnement dans ce texte ne génèrent pas tout seuls leur signification, taillée sur mesure pour une trame narrative particulière, mais dépendent plutôt de ce qui les précède. Leur substance interprétative est intertextuelle et non pas individualisée. Il ne suffit pas d'analyser l'environnement tel qu'il se présente seulement au Cuer ; il faut mesurer les liens de deux textes qui communiquent à travers presque trois siècles. Là aussi, René signale qu'il déjoue les principes opératoires de ses prédécesseurs pour commenter leurs œuvres, et par là, surtout, son monde à lui.

Un autre épisode météorologique chez René - cette fois, une tempête en mer confirme l'impression que l'auteur dépeint un monde qui n'épouse pas les désirs ou la psychologie des personnages. Ce monde n'est ni l'allié ni l'ennemi de l'être humain ; il lui est indifférent. Lorsque le Cuer et ses compagnons quittent la rive où ils sont venus trouver une barque pour les mener à l'Ile d'Amour, la mer est sereine, la brise douce, et rien ne laisse penser que leur voyage jusqu'à l'île ne se déroulera pas dans de bonnes conditions. Comme les voyageurs ne sont pas responsables de leur propre locomotion - deux gentes demoiselles rament pour eux - ils s'endorment, confiants. La passivité de ces chevaliers pourtant en quête signale au lecteur les limites inhérentes au quotidien d'un idéal : aucune victoire ultime chevaleresque n'est à prévoir lorsque la conduite des personnages ne correspond pas au code censé régir leurs actions. Quant au temps et au sommeil des compagnons, tout va bien jusqu'à ce que «le vent se [renforçe] si fort que les undes [...] [commencent] a croistre et la mer a grossir »; le trio d'hommes se réveille étourdis et déjà souffrant du mal de mer (René d'Anjou, 2003, p. 254). Ils sont harassés « pour la tormente de la mer qui [les traveille] sans repos » (René d'Anjou, 2003, p. 254). Cherchant à soulager leurs maux, les deux marinières les font (re)coucher (René d'Anjou, 2003, p. 257) ; les voilà de nouveau dans une posture bien vulnérable, blêmes et angoissés. Ballottés, ils ne peuvent faire autrement que d'attendre que la mer se calme. Ils ne sont pas maîtres de leur destin, et ne jouissent pas d'une protection divine spéciale. L'épreuve s'étend sur une journée entière : «Ainsi se passa le jour jusqu'à la nuit » (René d'Anjou, 2003, p. 257). Il faut prendre son mal en patience, et accepter que la mer houleuse ne cessera de se soulever que quand elle le voudra bien. Le rythme du temps - l'alternance de la lumière solaire et de l'obscurité - est immuable, et le Cuer et ses amis y voient l'ordre 
des choses. Ils n'interrogent pas le cycle diurne. Le temps météorologique, par contre, est changeant et imprévisible. Mais il est tout aussi externe aux personnages du récit que le lever et le coucher du soleil, et rappelle aux êtres humains leur incapacité à contrôler leur environnement. Les hommes subissent ce que crée la nature.

L'œuvre de René d'Anjou défait les topoï littéraires dès qu'il les met en œuvre : Le Cuer d'amours espris est un roman d'aventure qui traite souvent de l'ennui, et un récit d'amour qui dépeint la solitude ultime de l'amant. L'auto-démantèlement du texte suggère, quant au portrait de l'environnement et du temps, que le réflexe interprétatif médiéval, l'élan allégorisant qui caractérise le Moyen Âge, ne peut régir le non-humain. Le paysage et le temps ne sont pas là pour servir le Cuer. René procède par l'ironie ou tout simplement par l'humour pour exposer cette vérité ; les nausées peu chevaleresques des trois compagnons de haute mer en témoignent. Les faiblesses de l'homme se révèlent, entre autres, lorsqu'on le voit tributaire des aléas météorologiques.

Ce que René d'Anjou suggère par des méthodes obliques et un contenu dépendant pour sa pleine articulation de l'intertextualité, Aymon de Montfaucon l'a abordé de manière plus directe, voire sobre. Son Debat d'entre le gris et le noir se sert de la météorologie comme outil littéraire d'organisation ou de structure, plutôt que de signification. L'averse de pluie et de grêle qui pousse le chevalier Gris à reprendre son chemin ne représente pas un commentaire sur son état d'âme ou son avenir. Le temps qu'il fait n'est pas le miroir de ses forces ou de ses désirs. Parfois la pluie n'est que la pluie. Dans La Chanson de Roland, la tempête violente, le soleil suspendu dans sa traversée du ciel, déclaraient la présence constante de Dieu comme trait d'union entre l'homme et le monde extérieur. Dans Le Chevalier au lion, l'orage provoqué par tout chevalier tentant l'épreuve de la fontaine posait une relation entre la nature et l'être humain que l'homme était en mesure de régir. Le Roman de la rose, lui, offrait un cadre hors du temps dans les deux sens, une fusion parfaite entre l'esprit d'un homme et sa manifestation en forme de verger verdoyant : un idéal pérenne qui rendait des vicissitudes météorologiques impossibles. Dans sa discrétion et sa lucidité, peut-être est-ce Aymon de Montfaucon, cet auteur moins connu du Moyen Âge finissant, qui nous persuade le mieux du bien-fondé de sa pensée. 


\section{BIBLIOGRAPHIE}

Buettner, U. (2020). Talking about the Weather: Roland Barthes, Everydayness, the Feeling of Being, and Poetics.Ecozon@European Journal of Literature, Culture and Environment, 11,27-42.

Camenisch, C. (2015). Endless cold: a seasonal reconstruction of temperature and precipitation in the Burgundian Low Countries during the $15^{\text {th }}$ century based on documentary evidence. Climate of the Past, 11, 1049-1066.

Chiari, S. (2017). Climate as Climax in Shakespeare's Plays. Shakespeare in Southern Africa: Journal of the Shakespeare Society of Southern Africa, 29, 1-15.

Classen, A. (2010). Consequences of Bad Weather in Medieval Literature from Apollonius of Tyre to Marguerite de Navarre's Heptaméron. Arcadia: Internationale Zeitschrift für Literaturwissenschaft, 45, 3-20.

Cohen, J.J. \& Duckert, L. (Eds.) (2015). Elemental Ecocriticism: Thinking with Earth, Air, Water, and Fire. Minneapolis : University of Minnesota Press.

Cohen, J.J. \& Duckert, L. (Eds.) (2017). Veer Ecology: A Companion for Environmental Thinking. Minneapolis : University of Minnesota Press.

Curtius, E.R. (1990). European Literature and the Latin Middle Ages. New Haven : Yale University Press.

D’Anjou, R. (2003). Le Livre du Cour d'amour épris ((Ed. \& Trans. F. Bouchet). Paris : Librairie Générale Française.

George, M.W. (2014). Adversarial Relationships between Humans and Weather in Medieval English Literature. Essays in Medieval Studies: Proceedings of the Illinois Medieval Association, 30, 67-81.

Lorris, G. de \& Meun, J. de (1992). Le Roman de la rose (Ed. \& Trans. A. Strubel). Paris : Librairie Générale Française.

Pearsall, D. \& Salter, E. (1971). Landscapes and Seasons of the Medieval World. Toronto : University of Toronto Press.

Portnoy, P. (2017). Weekly Weather from the Anglo-Saxon Psalter: Rainfall and Dewfall in Picture and Prayer. In C. Biggam, C. Hough \& D. Izdebska (Eds.), The Daily Lives of the Anglo-Saxons (pp. 219238). Tempe, AZ : Arizona Center for Medieval and Renaissance Studies.

Short, I. (Ed. \& Trans.) (1990). La Chanson de Roland. Paris : Librairie Générale Française.

Troyes, Ch. de (1994). Le Chevalier au lion (Ed. \& Trans. D.F. Hult). Paris : Librairie Générale Française. Ungelenk, J. (2018). Literature and Weather: Shakespeare - Goethe - Zola. Berlin : De Gruyter. 